\title{
Scope of nanotechnology in ovarian cancer therapeutics
}

\author{
Murali M Yallapu', Meena Jaggi ${ }^{1,2,3}$, Subhash C Chauhan ${ }^{1,2,3^{*}}$
}

\begin{abstract}
This review describes the use of polymer micelle nanotechnology based chemotherapies for ovarian cancer. While various chemotherapeutic agents can be utilized to improve the survival rate of patients with ovarian cancer, their distribution throughout the entire body results in high normal organ toxicity. Polymer micelle nanotechnology aims to improve the therapeutic efficacy of anti-cancer drugs while minimizing the side effects. Herein, different types of polymer micelle technology based nanotherapies such as PLGA, polymerosomes, acid cleavable, thermosensitive, pH sensitive, and cross-linked micelles are introduced and structural differences are explained. Additionally, production methods, stability, sustainability, drug incorporation and drug release profiles of various polymer micelle based nanoformulations are discussed. An important feature of polymer micelle nanotechnology is the small size (10-100 nm) of particles which improves circulation and enables superior accumulation of the therapeutic drugs at the tumor sites. This review provides a comprehensive evaluation of different types of polymer micelles and their implications in ovarian cancer therapeutics.
\end{abstract}

\section{Introduction}

Ovarian cancer is the fifth most prevalent cancer among women with a life time risk of 1.4 to $1.8 \%$ for women living in the US. There are no early symptoms for ovarian cancer which hinders detection until it reaches advanced stages. Survival of the patients is primarily dependent on the disease stage of the patients. For example, stage I, II, III, and IV ovarian cancer have median 5-year survival rates of approximately 93\%, 70\%, $37 \%$, and $25 \%$, respectively $[1,2]$. Diagnosed ovarian cancers can be treated by eliminating the cancerous tissue through surgery and care must be taken to prevent the disease from recurring. Surgery alone is effective for only stage I disease, whereas chemotherapy is required in all other stages of ovarian cancer [3]. Therefore, our current review article is focused on the concept of improving the efficacy of ovarian cancer therapeutics using polymer micelle nanotechnology approaches.

Chemotherapy Agents used for Ovarian Cancer Treatment Chemotherapy helps to improve the overall survival of patients with ovarian cancer. Many chemotherapeutic

\footnotetext{
* Correspondence: Subhash.Chauhan@usd.edu

${ }^{1}$ Cancer Biology Research Center, Sanford Research/USD, Sioux Falls, SD 57104, USA

Full list of author information is available at the end of the article
}

agents (anti-cancer drugs) are available, including cisplatin (CP), paclitaxel (PTX), doxorubicin (DOX), decitabine (DB), gemcitabine, and their combinations for ovarian cancer treatment. There is significant interest in identifying novel therapeutic agents and improving the efficacy of existing therapeutic modalities. A number of randomized trials treating advanced ovarian cancer using a combination chemotherapy with HEXA-CAF (hexamethyl melamine (HMMA), cyclophosphamide (CPP), methotrexate (MTX) and fluorouracil (FU)) have achieved higher survival rates than using a single therapeutic agent [4]. Other clinical studies using cisplatin, adriamycin, and cyclophosphamide were initiated for stage III and IV ovarian cancer [5-8]. Nevertheless, these trials have not shown a significant benefit of one type of chemotherapy over another. Cisplatin and carboplatin (CBP) have been the most effective chemotherapeutic regimens for more than two decades $[9,10]$. The majority of current treatment approaches use platinumcontaining compounds such as cisplatin, oxaliplatin and transplatin [11-14]. Additionally, paclitaxel (Taxol, TAX) has been recognized as the most efficient chemotherapeutic agent for relapsed ovarian cancer [15]. Doxorubicin, in the form of doxorubicin $\mathrm{HCl}$ liposome injection (Doxil or Adiramycin ${ }^{\circ}$ ), has also been considered to be an effective therapeutic agent for many years [16]. 
A combination of doxorubicin, cyclophosphamide, and cisplatin resulted in an increase of $6 \%$ in the survival rate of patients versus a treatment of only cyclophosphamide or cisplatin [17]. Gemcitabine has shown positive response in patients who are resistant to cisplatin therapy and it is also a well tolerated drug [18]. An overall $74 \%$ response rate was achieved using a combination of gemcitabine with platinum (Gemplat or GemCis) for recurrent carcinoma [19-22]. A flow chart outlining the management of chemotherapeutic strategies is provided in the Figure 1.

It is evident from Figure 1 that, most of the chemotherapeutic drugs have demonstrated a significant therapeutic outcome, at the same time they exhibited adverse side effects [23]. Further, they are not effective in treating the recurrence of ovarian cancer. Importantly, ovarian cancer patients are often initially responsive to these therapeutic modalities but eventually become resistant to therapy. Therefore, drug resistance remains the major obstacle in ovarian cancer treatment. One way to improve the efficacy and specificity of chemotherapeutic agents is through nanotechnology based formulations (encapsulated, conjugated, or entrapped/ loaded forms in nanocarriers or drug delivery vehicle/ vectors). Nanotechnology mediated therapies promote a controlled delivery of chemotherapeutic drug(s) in a targeted way which directly acts on the cancer site for prolonged periods of time with minimal or no normal organ toxicity. Therefore, this review will focus on the design of nanotechnology formulations for ovarian cancers treatment.

\section{Nanotechnology in Drug Delivery}

Chemotherapeutic agents in solution or polymer solution which are delivered orally or intravenously have poor pharmacokinetics with a narrow therapeutic window (Figure 2A). These agents reach a maximum tolerated concentration immediately and then are eliminated from the blood. An ideal drug formulation with maximum benefits for patients should release at a minimum effective concentration over a period of time. Nanotechnology promises to play an important role in satisfying these aspects as a drug delivery carrier/vector (Figure 2B). Nanotechnology based drug carriers such as

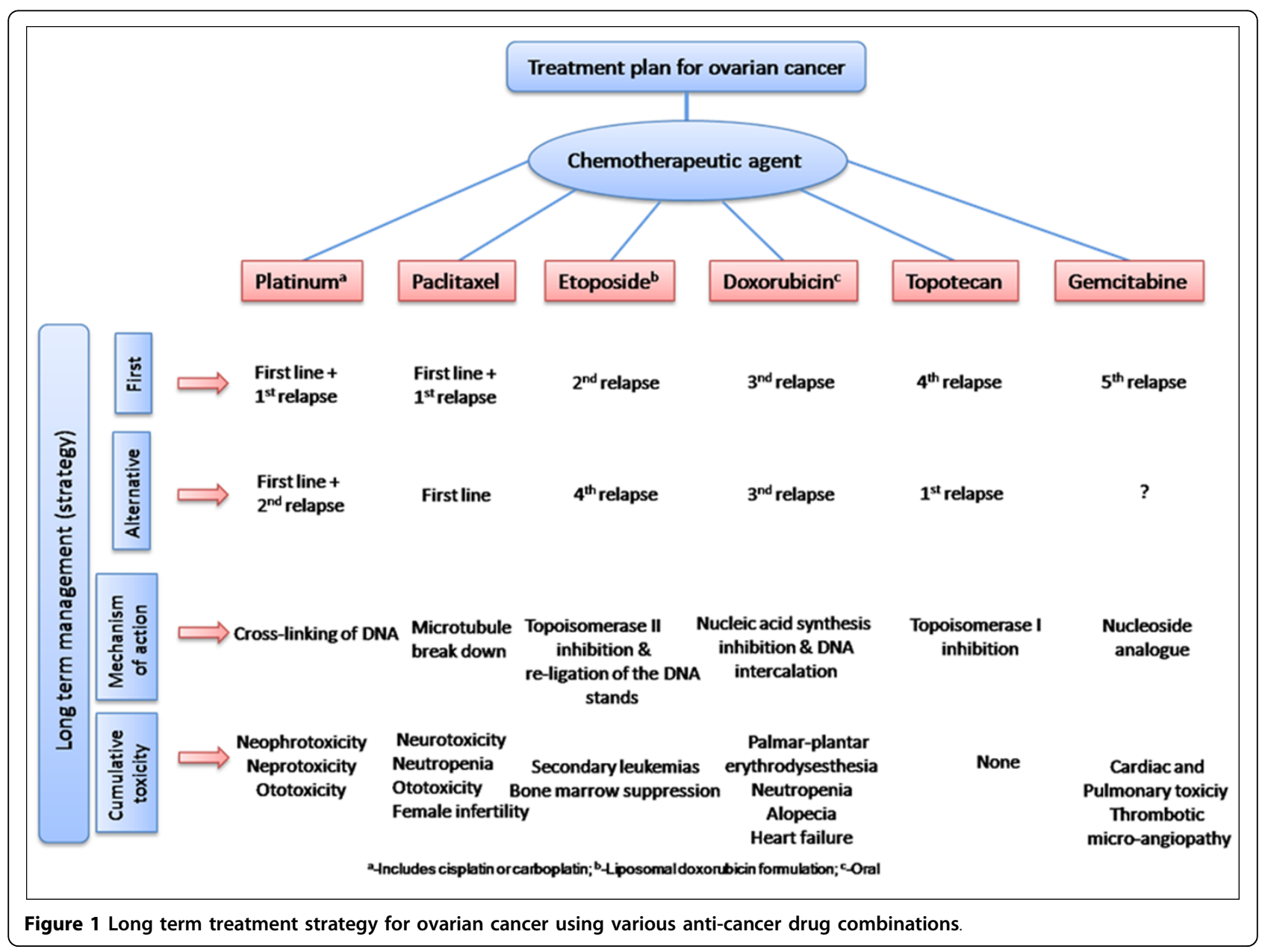




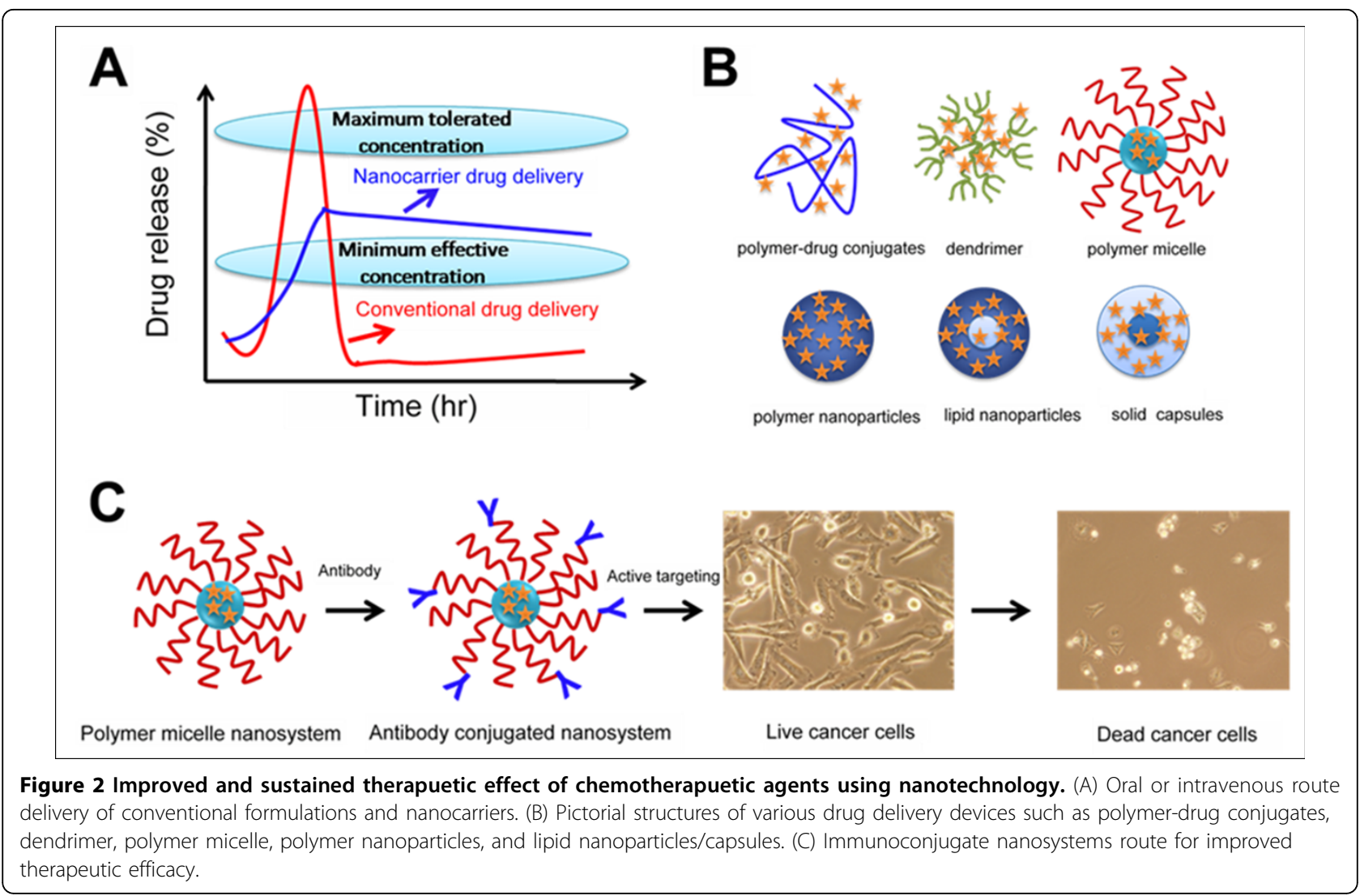

polymer-drug conjugates, dendrimer, polymer micelles, carbon nanotubes, lipid/solid nanoparticles, and polymer nanoparticles have numerous benefits over conventional methods. Nanotechnology based therapeutics have been proven to improve drug efficacy, reduce toxicity in healthy tissue, and improve patient compliance. Many of these nanoparticles are currently in use for cancer therapies [24]. A list of clinical and preclinical trials of these nanotechnology based formulations have been reviewed by Quan et al. [25]. The design of a universal nanotechnology formulation with chemotherapeutic agents is extremely crucial. A successful formulation, one that acts as a good therapeutic carrier for the cancer therapies, would exhibit the following features: (a) stable in the physiological environment, (b) longer circulation life time (c) avoid opsonization and reticuloendothelial system (RES) process, (d) promote endocytosis, and (e) enhance tumor uptake. The specificity of these formulations can be further enhanced by the conjugation of antibodies to the nanoformulations and these immunoconjugated formulations will have a better therapeutic efficacy over other drug formulations (Figure 2C).

\section{Drug Delivery Approach}

Chemotherapeutic agent(s) or anti-cancer drug(s) delivery to tumors can be achieved by either a passive or an active mechanism. These mechanisms are shown in a pictorial representation in Figure 3. The passive targeting takes place through the diffusion into tumors or angiogenic tumor vasculatures which have leaky vessels with smaller gaps of $100-2000 \mathrm{~nm}$. The nanoformulations (drug loaded nanoparticles) have more interstitial access to the tumor and enhance the retention in tumors. The leaky vasculature promotes the uptake of nanoformulations by the tumors, which become entrapped inside, and due to impaired and poor lymphatic drainage, promotes Enhanced Permeation and Retention (EPR) index. In addition, the size and charge of nanoparticles dictates the passive targeting to the tumors [25-28]. In comparison, the active targeting mode utilizes the conjugation of nanoparticles to immunogens (antibodies or targeting moieties). Delivery of drugs can be improved through tumor specific antibody conjugated nanoparticle system (active targeting) over simple drug loaded nanoparticle system (passive targeting). First, the transport of nanoparticles uptake by the tumor site is increased by longer circulation as a result of the EPR effect. Secondly, the targeting moiety assists in endocytosis of nanoparticles which, in general, increases internalization of nanoparticles for an improved therapeutic effect $[29,30]$. This targeting approach is promising which has shown enhanced therapeutic effects in animal models via substantial 


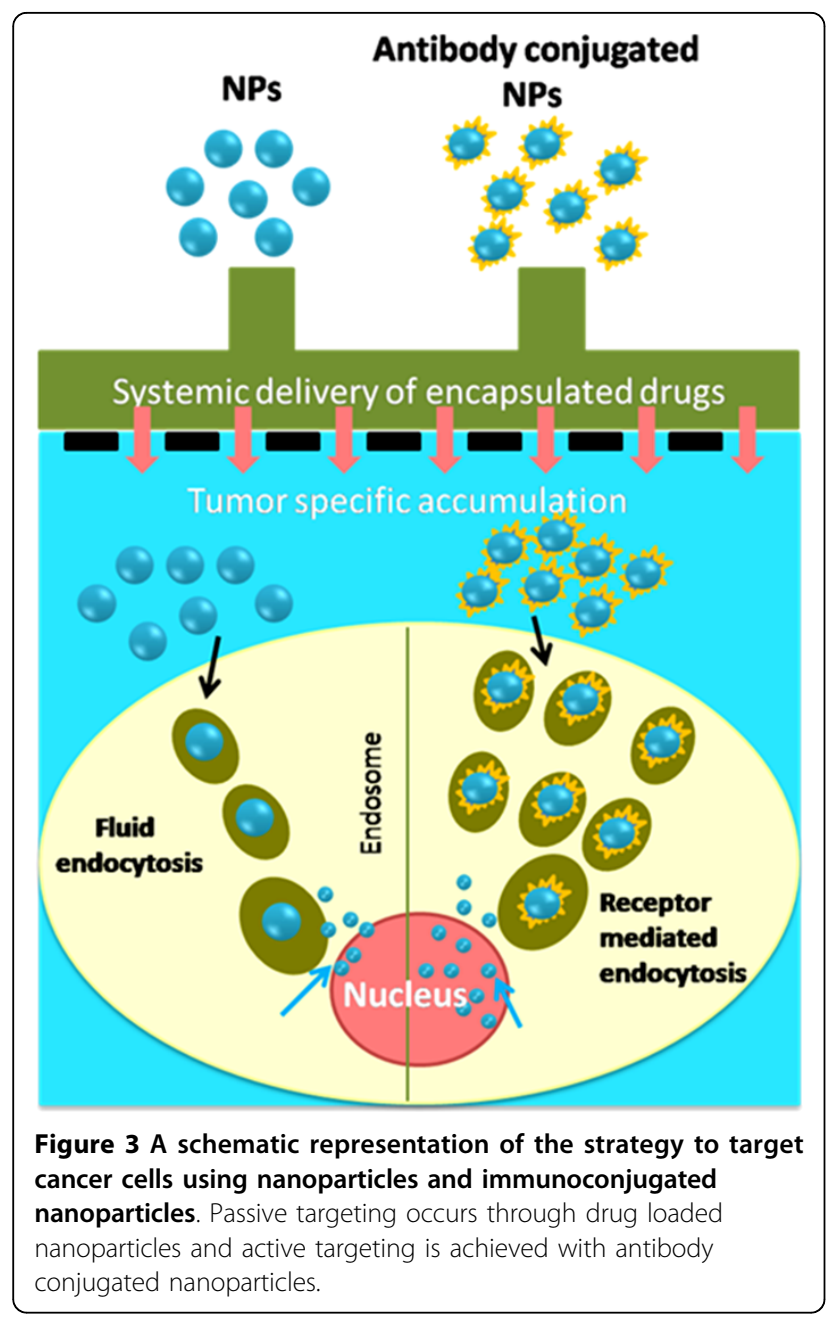

increase in nanoparticles internalization in cancer cells $[31,32]$. In addition to anti-cancer drug delivery, the internalization of nanoparticles is also an important factor in gene, siRNA, DNA and biomacromolecular delivery. Therefore, combination of controlled and targeted delivery improves the efficacy of delivering drugs, genes and biomolecules. In this review we focused on current nanoformulations, especially on polymer micelle nanosystems which have been recognized for their special characteristics.

\section{Polymer Micelle Nanotechnology}

Polymer micelle nanotechnology based delivery of chemotherapeutic agents, imaging agents, biomacromolecules and radionuclides in a tumor-targeted way may enhance diagnosis as well as the outcome of cancer therapy [33]. In this direction, a few clinical trials of various polymer micelle nanotechnology therapies are in the development stage [34]. To describe simply, polymer micelles are formed by a hydrophobic core layered with hydrophilic chains through a spontaneous self-assembly of block or graft copolymers [35,36]. The primary function of polymer micelles is to protect and improve the solubility and stability of hydrophobic (lipophilic) drugs. It has been shown that the aqueous solubility can be increased up to 30,000 -fold $[37,38]$. In our studies, we have demonstrated that $\beta$-cyclodextrin, poly ( $\beta$-cyclodextrin), and polymer nanoparticle curcumin assemblies can improve stability of curcumin by 6-8 fold [39-41]. This higher stability occurs when the drug molecules partition into the hydrophobic core of the polymer micelles and a protective hydrophilic shell interface acts as external medium.

There are a number of hydrophobic core-forming biocompatible and biodegradable polymer micelles, such as, poly(ethylene-co-propylene-co-ethylene oxide) (PEO$b$-PPO-b-PPO) or poly(ethylene-co-propylene oxide) (PEO- $b$-PPO), poly(lactic acid) (PLA), poly $(\mathrm{D}, \mathrm{L}$-lactide) (PDLLA), poly(lactic-co-glycolic acid) (PLGA), poly ( $\varepsilon$ caprolactone) (PCL), poly(hydroxybutyrate) (PHB), and poly(beta-benzyl L-asparate) [42-47] being used in drug delivery applications. The formation of micelles of these polymers is feasible only at a specific concentration (i.e., critical micelle concentration, CMC). The polymer micelle with a lower CMC value is a better choice for these applications. Figure 4 schematically presents different models of formation of polymer micelles based on their self-assembly mechanisms.

\section{Conventional Polymer Micelles}

A number of natural or synthetic di-block or tri-block copolymers which are biodegradable/biocompatible in nature, have been utilized to load various drugs/biological molecules. Among them, poly(lactic-co-glycolic acid) (PLGA) generated micelles are well known. In addition, the parent PLGA polymer is FDA approved for use in industry and medicine. Structurally varied nanoformulations, such as comb-like amphiphilic PLGA- $b$-poly(ethylene glycol) methacrylate (PLGA- $b$-PEGMA) copolymer, PLGA- $b$-poly (ethylene glycol)- $b$-PLGA (PLGA- $b$-PEG$b$-PLGA) tri-block copolymer, three-arm and four-arm star-shaped PLGA- $b$-PEG block copolymer micelles are available for drug delivery applications [48-50]. In addition, Park et al. [51] recently developed a surface crosslinking PLGA- $b$-PEG copolymer to improve the overall stability of polymer micelles utilizing a shell layer of vinyl pyrrolidone. A natural carbohydrate polymer (i.e. hyaluronic acid (HA) copolymer) can be utilized as target specific micelle carriers for doxorubicin (DOX) by conjugating to PLGA polymer [52]. This formulation allowed loading of 4.8-7.2 wt.\% DOX (i.e., DOX-HA-gPLGA) which exhibited 5.2-fold greater cytotoxicity in the cancer cells over free DOX ( $\mathrm{IC}_{50}$ value of DOX-HA$g$-PLGA $=0.67 \mathrm{mg} \cdot \mathrm{mL}^{-1}$ and free DOX $=3.48 \mathrm{mg} \cdot \mathrm{mL}^{-1}$ ). Similarly, a mixed micelle nanoformulation of DOX 

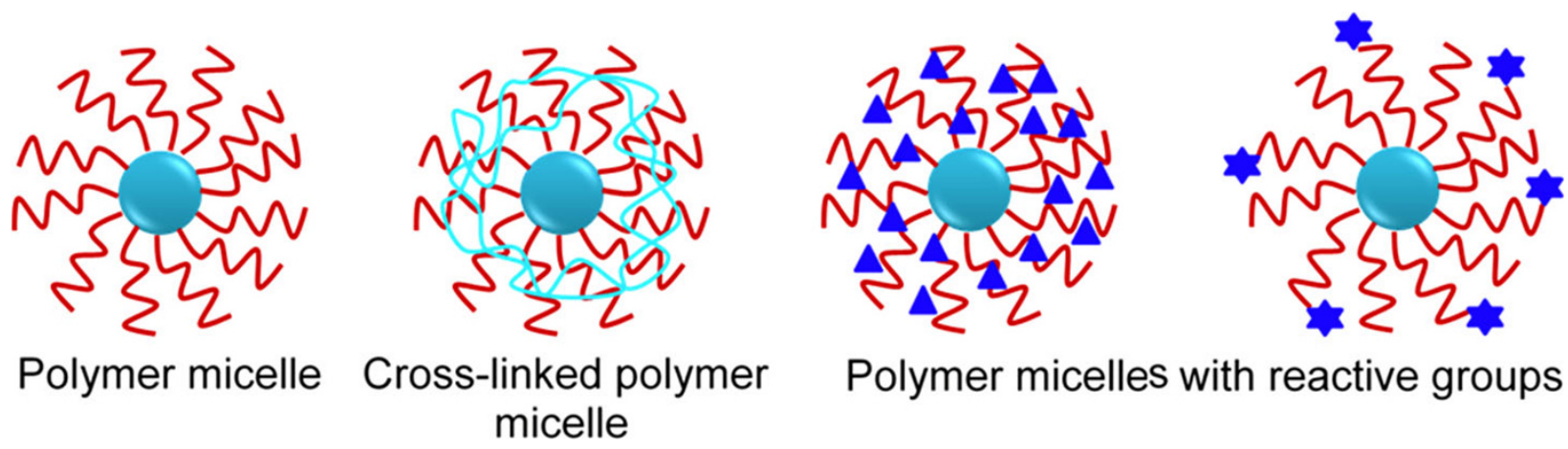

Polymer micelles with reactive groups

Figure 4 Different types of polymer micelle formations through the self-assembly process. This process is always favored by hydrophobichydrophobic interactions within the block copolymers. The core is completely hydrophobic which can be used to load anti-cancer drugs. Reactive functional groups can be utilized for antibody conjugations. Illustrations are based on their chemical structures.

loaded TPGS/PLGA- $b$-PEG- $b$-FOL (TPGS $=\alpha-$ tocopheryl succinate esterified to polyethylene glycol 1000 and $\mathrm{FOL}=$ folate) has shown higher cellular uptake of DOX, which resulted a higher degree of apoptosis in drug-resistant cancer cells. Nanoformulation of PLGA coated with poly(L-lysine)-PEG-folate conjugates has shown an enhanced cellular uptake via folate receptor-mediated intracellular delivery [53]. Our data also suggest that PLGA formulations combined with poly (vinyl alcohol) (PVA) achieved intracellular uptake and exhibited improved therapeutic effects of curcumin in cisplatin resistant ovarian $(\mathrm{A} 2780 \mathrm{CP})$ and metastatic breast (MDA-MB-231) cancer cells (Figure 5) [41].

\section{Other Type of Polymer Micelle Nanoparticles}

All drug delivery carrier properties are determined by their stability, solubility, surface charge and type of functional groups which facilitate the encapsulated drug release and targeting characteristics to tumor cells. Pluronic polymers (i.e., poly(ethylene oxide)- $b$-poly(propylene oxide)- $b$-poly(ethylene oxide or PEO- $b$-PPO- $b$ $\mathrm{PEO})$ are known as easily forming micelle drug carriers with a $40 \mathrm{~nm}$ diameter. These micelle nanocarriers have the ability to increase the solubility of various hydrophobic anti-cancer drugs as well as enable passive targeting to the solid tumor. Studies have also demonstrated that pluronic micelles promote enhanced cytotoxic activities of various anti-cancer drugs by sensitization of cancer cells attributed to the inhibition of P-glycoprotein (P-gp) activity by depletion of adenosine-5'-triphosphate (ATP) [54]. Poly(ethylene oxide)-linked poly(ethylene imine) (PEO- $l$-PEI) micelle gel is a good example which binds oligonucleotide (ODN) molecules and its delivery is enhanced through receptor-mediated delivery. In general, ODNs are useful therapeutic agents which suffer from severe enzymatic degradation by nucleases. Encapsulation of ODNs in PEG/PEI micelles not only regulated the growth of ovarian cancer cells (A2780) but also lowered ODN concentrations and resulted in significant tumor growth suppression in vivo [54,55]. Similarly, stable micelle formulations of 5'-triphosphates of cytarabine (araCTP), gemcitabine ( $\mathrm{dFdCTP}$ ), and floxuridine (FdUTP) in PEG- $l$-PEI networks have been proven to accumulate faster and inhibit tumor growth in vivo [56]. Curcumin-casein micelle complexes not only exhibited higher cytotoxicity against HeLa cells but were also capable of damaging cell nucleus as a result of apoptosis at a concentration of $30 \mu \mathrm{M}$ curcumin [57]. These complexes were also more efficiently internalized in the cells. In our recent investigations $[39,40]$, we have proven that the natural anti-cancer and cancer prevention agent, curcumin, is effective in therapies with self-assembly or nano self-assembly formulations of $\beta$-cyclodextrin or poly( $\beta$-cyclodextrin).

PEG/PDLLA-Taxol combination (Genexol $\left.{ }^{\circ}-\mathrm{PM}\right)$ is a formulation with high anti-tumor efficacy in human ovarian cancer cell line (OVCAR-3) [58]. In another report, triptolide (TP) loaded PDLLA/PEG nanocarrier was shown to significantly inhibit tumor growth via i.v. injections at the dose levels of $0.0375,0.075$ and 0.15 $\mathrm{mg} / \mathrm{kg}$, and their inhibition rates were $42.5 \%, 46.0 \%$ and $49.9 \%$, respectively. Hydrolyzable polyesters of PCL and PDLLA are useful formulations to encapsulate paclitaxel, ellipticine, and doxorubicin drugs [59-61]. Further, a novel poly(ethyl ethylene phosphate) (PPE, polyphosphorus ether) and PCL biodegradable triblock copolymer micelles were developed as drug carriers [62]. These micelles are biodegradable, cytocompatible, small sized particles and show improved drug loading efficiency with an increase of PPE molecular weight. The advanced features of these micelles result in more flexibility and their physico-chemical properties can be adjusted through changing the side group conjugation to phosphorus [63]. Another biocompatible micelle (i.e., poly[2-(methacryloyloxy)ethyl phosphorylcholine] or MPC) conjugated with folate targeting moiety to poly 


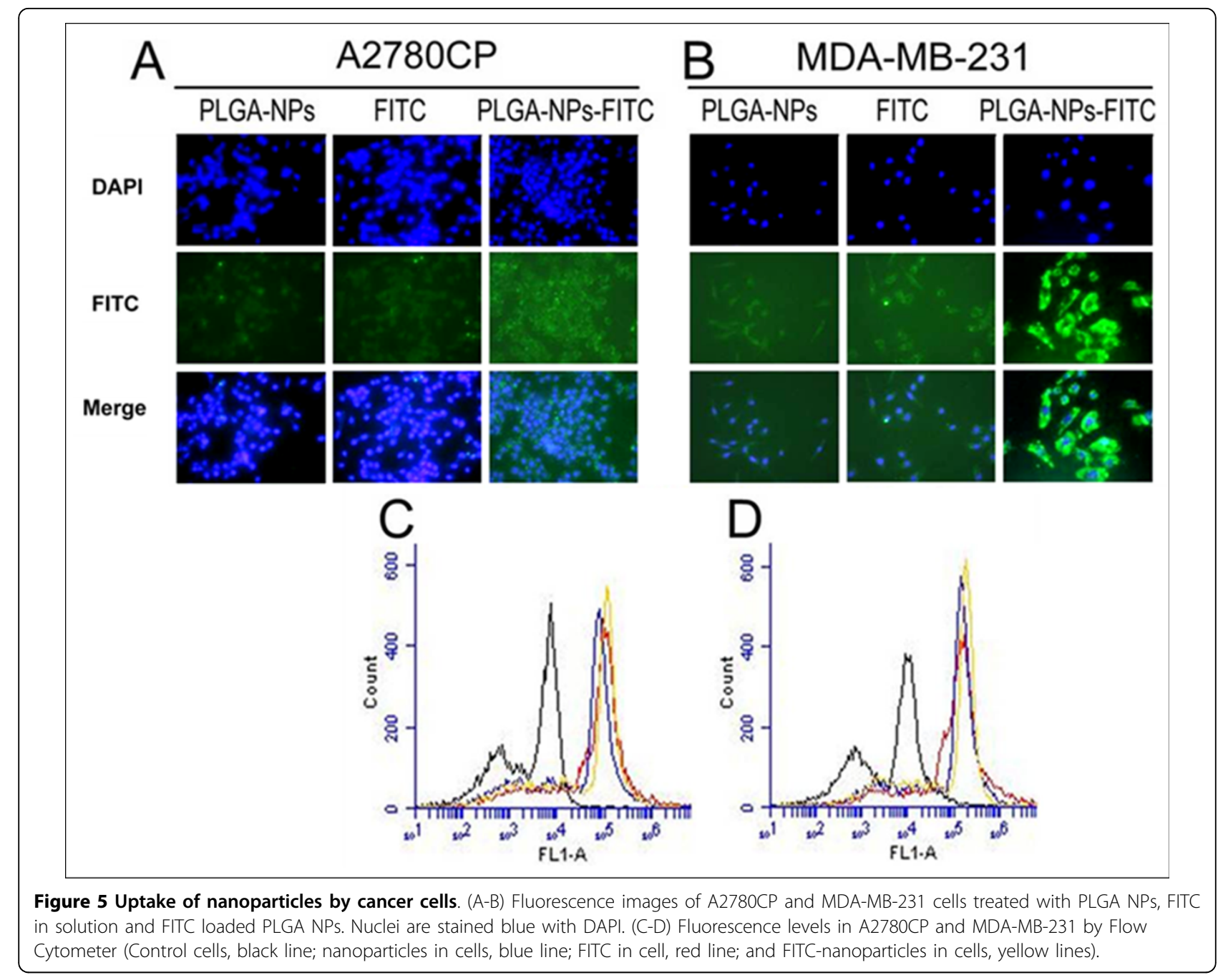

[2-(diisopropylamino)ethyl methacrylate] (DPA) (i.e., MPC-DPA-FA), demonstrated a 2.5 -fold increase in tamoxifen and paclitaxel uptake [64]. Additionally, cationic polymer micelles can be effectively mediated through endosomal rupture or degradation (i.e., "proton sponge" effect) but often failed at in vivo studies due to rapid clearance from the circulation. Therefore, polymerosomes were developed which are cationic polymer micelles that shield the positive charge with a neutral polymer (such as PEG) coating. For example, polyelectrolyte complex (PEC) micelles with luteinizing hormone-releasing hormone (LHRH) peptide exhibited enhanced cellular uptake by increasing VEGF siRNA gene silencing efficiency via receptor-mediated endocytosis compared with those without LHRH on LHRH receptor overexpressing ovarian cancer cells (A2780) [65]. Epidermal growth factor (EGF)-conjugated MePEG- $b$-PCL micelles can be delivered at a concentration 13 times more potent than free EGF [66].

\section{pH Sensitive and Acid Cleavable Polymer Micelle Nanoparticles}

The main advantage of these micelles is that encapsulated drugs are burst release in the acidic intracellular compartments such as endosomes or lysosomes. These formulations improved anti-tumor activity through intracellular pH-sensitive drug delivery [67]. Additionally, their folate conjugation was proven to enhance in vivo anti-tumor efficacy at lower effective doses [68]. Furthermore, $\mathrm{pH}$-sensitive micelles poly(L-hystidine)- $b$-PEG and PLA- $b$-PEG- $l$-FOL (PHSM-f) were superior compared to free and conventional polymer micelles [69]. The in vivo experiments using a sensitive micelle system also demonstrate accumulation of particles at the tumor site and tumor regression was 4-5 fold greater than free DOX after 27 days from the first i.v. injection. One study noted that the half-life of DOX in the $\mathrm{pH}$ sensitive micelles increased about 6-fold from free DOX in PBS and plasma media. Their uptake at pH 6.8 was 5 times more than at 
$\mathrm{pH} 7.4$, indicating that the drug release triggered by the reduced tumor $\mathrm{pH}$ was effective after the micelles were accumulated by the EPR effect. Another novel tetrablock copolymer [poly(ethylene glycol)- $b$-poly(L-histidine)- $b$-poly(L-lactic acid)- $b$-poly(ethylene glycol)] is capable of triggering release of DOX at pH 6.8 (i.e., tumor acidic $\mathrm{pH}$ ) or $\mathrm{pH} 6.4$ (i.e., endosomal $\mathrm{pH}$ ) compared to normal $\mathrm{pH} 7.4$ [70]. This triggering or burst release effect is dependent upon the molecular weight of the PLA block existing in the tetra polymer which could be a successful therapy for treating solid cancers or delivering cytoplasmic cargo in vivo. A new formulation composed of DOX in PDLLA- $b$-PEG-b-poly(L-histidine)TAT (transactivator of transcription) micelle was able to expose TAT only at a slightly acidic tumor extracellular $\mathrm{pH}$ to facilitate the internalization process [71]. These micelles were tested with the xenograft models of human ovarian tumor drug-resistant A2780/AD, human breast tumor drug-sensitive MCF-7 and human lung tumor A549 in a nude mice model, and all tumors considerably regressed in size after three bolus injections at a dose of $10 \mathrm{mg}$ DOX per kg body weight, at three day intervals, while minimum weight loss was observed. The conjugation of drugs to the acid cleavable micelle polymers facilitated prolonged release of drugs [72]. Doxorubicinconjugated PLLA-mPEG micelles were more potent because they were taken up within cells with simultaneous rapid release of cleaved doxorubicin into the cytoplasm from acidic endosomes [72]. A Triblock copolymer conjugated with DOX through the end $\mathrm{OH}$ groups of copolymers, indicated that hydrazone linkage was cleaved under acidic conditions [73]. This behavior was confirmed by flow cytometry and confocal microscopy which demonstrated the extent of cellular uptake of micelle conjugated DOX and distribution in the cytoplasm, endosomal/liposomal vesicles, and nucleus, while the free drug was localized within the nucleus.

\section{Cross-linked Polymer Micelle Nanoparticles}

Various polymer micelle nanoparticles can control the triggered release of the active therapeutic agents, but most of these polymer micelle nanoparticles have drawbacks as delivery carriers. For example, paclitaxel was readily disassociated from the micelle nanoparticle just after injection into the blood stream [74]. This dissociation may be due to the decomposition of micelles $\alpha$ - and $\beta$-globulins and translocation of paclitaxel to the abundant lipid components and carriers in blood [75]. To address this drawback of polymer micelle nanoparticles, a possible strategy is to design cross-linked biodegradable micelles [76]. These micelles can shield drug molecules tightly by the cross-linked corona and biodegradable cross-linking releases the drug from micelles in a controlled manner. Core micelle cross-linked with divalent metal cations display high stability but also exhibit $\mathrm{pH}$ dependant swelling/collapse behavior [77]. These systems have remarkably high platinum loading efficiency (i.e., $\sim 22 \%$ wt./wt.) and exhibited slow release of platinum in a sustained manner from the cisplatin-loaded crosslinked micelles in physiological saline. A new formulation based on $\alpha$-methoxypoly(ethylene oxide)- $b$-poly[ $N$ (3-aminopropyl)methacrylamide]-b-poly[2-(diisopropylamino)ethyl methacrylate] (mPEO-PAPMA-PDPAEMA), poly( $N$-isopropylacrylamide), and $N$-hydroxysuccinimidyl esters (NHS) prevented the dissolution of micelles due to dilution effects and enabled $\mathrm{pH}$ sensitive and potentially cleavable sites for micelle disassembly [78].

\section{Novel Polymer Micelle Nanotechnology Strategies}

Double-hydrophilic block copolymer based micelles have more external hydrophilic behavior which mimics biological fluid, unlike core-shell block copolymers $[79,80]$. The first hydrophilic charged block copolymer binds to the chemotherapeutic agent and the second hydrophilic block allows for steric stabilization. Polyamino

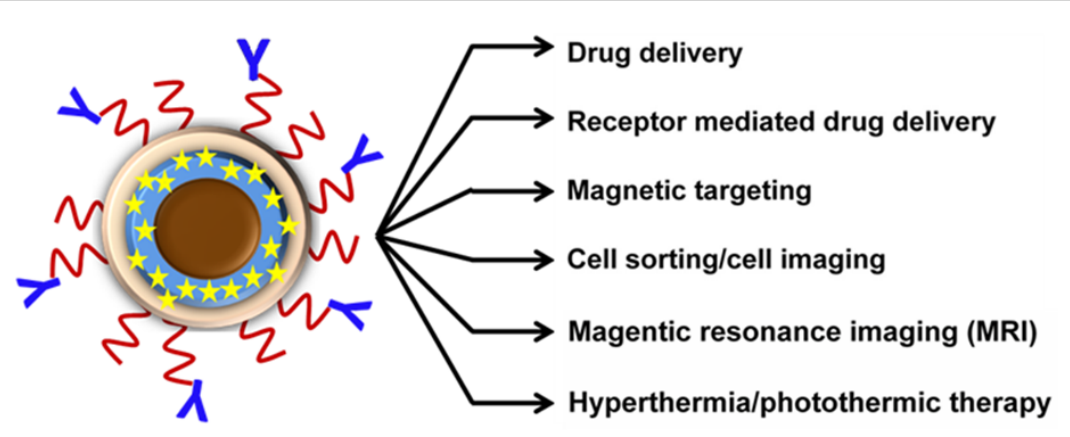

Multi-functional magnetic nanoparticles

Figure 6 Multi-functional magnetic nanoformulation with curcumin/photo activator loaded, double layer, antibody conjugation for various medical applications. 
acid-b-polyethylene oxide copolymers are excellent examples which can bind the oppositely charged species such as drugs (cisplatin and doxorubicin), proteins or peptides, nucleic acids, and lysozymes [81-84]. Ultrasound sensitive polymer micelle nanoparticles are another modality of drug targeting to tumors by a localized release. These micelles are degraded when subjected to ultrasound into unimers which enhances the cell membrane perturbation [85]. Even a short exposure of 15 to 30 seconds to high-frequency ultrasound leads to a significant increase in the intracellular DOX uptake from pluronic micelles [86].

Magnetic nanoparticle based micelles act as drug carriers as well as external magnetic field guides in cancer therapy treatments [87]. Developing micelle-magnetic nanoparticles is a promising alternative. Recently, such formulations were developed to gain different biological functions while using only one formulation [88]. These formulations can be applied not only for drug delivery techniques but also magnetic resonance imaging (MRI), visible targeting, magnetically targeted photodynamic therapy, targeted thermo-sensitive chemotherapy, and luminescence/near-infrared/multi-model imaging applications [89-96]. In this regard, one novel formulation composed of an iron oxide nano-core stabilized with a multi-layer coating could achieve better feasibility in drug delivery, imaging and hyperthermia properties. However, the higher hydrodynamic diameter $(>200 \mathrm{~nm})$ in aqueous medium limits its use in cancer therapeutic applications [97]. Therefore, we have been developing a novel formulation of magnetic nanoparticles composed of iron oxide core that is subsequently coated with $\beta$-cyclodextrin (CD) and pluronic F127 polymer (F-127) which possesses anti-cancer drug loading and antibody conjugation features and can be utilized for multifunctional applications (Figure 6). The advantages of this formulation include smaller particle size, relatively lower protein binding, higher drug loading efficiency and enhanced particles uptake in cancer cells without hampering inherent magnetization characteristics.

\section{Conclusions}

Polymer micelle nanotechnology has demonstrated that nanoparticles are capable of loading anti-cancer drugs which can be specifically targeted to tumors through the conjugation of tumor specific antibody/moiety. Multifunctional polymer micelles, including nanogels/magnetic based micelles, possess characteristics which could improve ovarian cancer therapy. These formulations have capabilities of MRI visible targeting, targeted photodynamic therapy, thermosensitive therapy and luminescence/near-infrared/multi-model imaging properties, which will allow tracking and monitoring of nanoformulations and accumulated drug(s) at the tumor site during the therapy procedure.

\section{Acknowledgements}

The authors thankfully acknowledge Cathy Christopherson for editorial assistance. We thank Dr. Diane Maher, Mara Ebeling and Mitch Ray Dobberpuhl for the valuable suggestions. This work was supported by grants from Sanford Research/USD, Department of Defense (DOD) (PC073887), Governor's Cancer 2010, and NIH RO1 (CA142736) awarded to SCC and Department of Defense (DOD) (PC073643) and Governor's Cancer 2010 grants awarded to MJ.

\section{Author details}

'Cancer Biology Research Center, Sanford Research/USD, Sioux Falls, SD 57104, USA. ${ }^{2}$ Department of Obstetrics and Gynecology, Sanford School of Medicine, The University of South Dakota, Sioux Falls, SD 57105, USA. ${ }^{3}$ Basic Biomedical Science Division, Sanford School of Medicine, The University of South Dakota, Sioux Falls, SD 57105, USA.

\section{Authors' contributions}

MMY drafted the manuscript. MJ and SCC participated in revising the manuscript. All authors have read and approved the final manuscript.

\section{Competing interests}

The authors declare that they have no competing interests.

Received: 16 July 2010 Accepted: 6 August 2010

Published: 6 August 2010

\section{References}

1. Jemal A, Siegel R, Ward E, Hao Y, Xu J, Thun MJ: Cancer statistics 2009. CA Cancer I Clin 2009, 59:225-249.

2. Chauhan SC, Kumar D, Jaggi M: Mucins in ovarian cancer diagnosis and therapy. J Ovarian Res 2009, 2:21.

3. Armstrong DK: Relapsed ovarian cancer: challenges and management strategies for a chronic disease. Oncologist 2002, 7(5):20-28.

4. Carmo-Pereira J, Costa FO, Henriques E, Ricardo JA: Advanced ovarian carcinoma: a prospective and randomized clinical trial of cyclophosphamide versus combination cytotoxic chemotherapy (HexaCAF). Cancer 1981, 48(9):1947-1951.

5. Ehrlich CE, Einhorn L, Stehman FB, Blessing J: Treatment of advanced epithelial ovarian cancer using cisplatin, adriamycin and cytoxan-the Indiana University experience. Clin Obstet Gynaecol 1983, 10(2):325-335.

6. Diaz-Rubio E, Escudero M, Martin-Jimenez M, Vidart JA, Gonzalez-Larriba JL, Herraiz MA, Lopez Vega JM, Bullon F: Treatment of advanced ovarian cancer with cisplatin, adriamycin and cyclophosphamide (PAC). Eur J Gynaecol Oncol 1989, 10(6):424-432.

7. Budd GT, Webster KD, Reimer RR, Martimbeau P, Livingston RB: Treatment of advanced ovarian cancer with cisplatin, adriamycin, and cyclophosphamide: effect of treatment and incidence of intracranial metastases. J Surg Oncol 1983, 24(3):192-195.

8. Bezwoda WR: Treatment of advanced ovarian cancer: a randomised trial comparing adriamycin or 4'epi-adriamycin in combination with cisplatin and cyclophosphamide. Med Pediatr Oncol 1986, 14(1):26-29.

9. Advanced Ovarian Cancer Trialists Group: Chemotherapy in advanced ovarian cancer: an overview of randomised clinical trials. BMJ 1991, 303(6807):884-893.

10. Aabo K, Adams M, Adnitt P, Alberts DS, Athanazziou A, Barley V, Bell DR Bianchi U, Bolis G, Brady MF, et al: Chemotherapy in advanced ovarian cancer: four systematic meta-analyses of individual patient data from 37 randomized trials. Br J Cancer 1998, 78(11):1479-1487.

11. Stewart L, Advanced Ovarian Cancer Trialists Group: Chemotherapy for advanced ovarian cancer. Cochrane Database of Systematic Reviews. Reviews 1999, 1:CD001418.

12. Misset IL, Bleiberg H, Sutherland W, Bekradda M, Cvitkovic E: Oxaliplatin clinical activity: a review. Crit Rev Oncol Hematol 2000, 35(2):75-93.

13. Ozols RF: Paclitaxel (Taxol)/carboplatin combination chemotherapy in the treatment of advanced ovarian cancer. Semin Oncol 2000, 3(7):3-7. 
14. Thigpen JT: Chemotherapy for advanced ovarian cancer: overview of randomized trials. Semin Oncol 2000, 3(7):11-16.

15. McGuire WP, Rowinsky EK, Rosenshein NB, Grumbine FC, Ettinger DS, Armstrong DK, Donehower RC: Taxol: a unique antineoplastic agent with significant activity in advanced ovarian epithelial neoplasms. Ann Intern Med 1989, 111(4):273-279.

16. A'Hern RP, Gore ME: Impact of doxorubicin on survival in advanced ovarian cancer. J Clin Oncol 1995, 13(3):726-732.

17. Cyclophosphamide plus cisplatin versus cyclophosphamide, doxorubicin and cisplatin chemotherapy of ovarian carcinoma: a meta-analysis. The Ovarian Cancer Meta-Analysis Project. J Clin Oncol 1991, 9(9):1668-1674.

18. Kaufmann M, von Minckwitz G: Gemcitabine in ovarian cancer: an overview of safety and efficacy. Eur J Cancer 1997, 33(1):S31-33.

19. Villella J, Marchetti D, Odunsi K, Rodabaugh K, Driscoll DL, Lele S: Response of combination platinum and gemcitabine chemotherapy for recurrent epithelial ovarian carcinoma. Gynecol Oncol 2004, 95(3):539-545.

20. Baetz T, Belch A, Couban S, Imrie K, Yau J, Myers R, Ding K, Paul N, Shepherd L, Iglesias J, et al: Gemcitabine, dexamethasone and cisplatin is an active and non-toxic chemotherapy regimen in relapsed or refractory Hodgkin's disease: a phase II study by the National Cancer Institute of Canada Clinical Trials Group. Ann Oncol 2003, 14(12):1762-1767.

21. Crump M, Baetz T, Couban S, Belch A, Marcellus D, Howson-Jan K, Imrie K, Myers R, Adams G, Ding K, et al: Gemcitabine, dexamethasone, and cisplatin in patients with recurrent or refractory aggressive histology Bcell non-Hodgkin lymphoma: a Phase II study by the National Cancer Institute of Canada Clinical Trials Group (NCIC-CTG). Cancer 2004, 101(8):1835-1842.

22. Gridelli C, Gallo C, Shepherd FA, Illiano A, Piantedosi F, Robbiati SF, Manzione L, Barbera S, Frontini L, Veltri E, et al: Gemcitabine plus vinorelbine compared with cisplatin plus vinorelbine or cisplatin plus gemcitabine for advanced non-small-cell lung cancer: a phase III trial of the Italian GEMVIN Investigators and the National Cancer Institute of Canada Clinical Trials Group. J Clin Oncol 2003, 21(16):3025-3034.

23. Dunton $\mathrm{CJ}$ : Management of treatment-related toxicity in advanced ovarian cancer. Oncologist 2002, 7(5):11-19.

24. Haley B, Frenkel E: Nanoparticles for drug delivery in cancer treatment. Urol Oncol 2008, 26(1):57-64.

25. Quan CY, Wei H, Sun YX, Cheng SX, Shen K, Gu ZW, Zhang XZ, Zhuo RX: Polyethyleneimine modified biocompatible poly( $\mathrm{N}$-isopropylacrylamide)based nanogels for drug delivery. J Nanosci Nanotechnol 2008, 8(5):2377-2384.

26. Diez $S$, Navarro $G$, de llarduya $C T$ : In vivo targeted gene delivery by cationic nanoparticles for treatment of hepatocellular carcinoma. J Gene Med 2008, 11(1):38-45.

27. Sortino S, Mazzaglia A, Monsu Scolaro L, Marino Merlo F, Valveri V, Sciortino MT: Nanoparticles of cationic amphiphilic cyclodextrins entangling anionic porphyrins as carrier-sensitizer system in photodynamic cancer therapy. Biomaterials 2006, 27(23):4256-4265.

28. Xiong MP, Bae Y, Fukushima S, Forrest ML, Nishiyama N, Kataoka K, Kwon GS: pH-responsive Multi-PEGylated dual cationic nanoparticles enable charge modulations for safe gene delivery. ChemMedChem 2007, 2(9):1321-1327.

29. Breunig M, Bauer S, Goepferich A: Polymers and nanoparticles: intelligent tools for intracellular targeting? Eur J Pharm Biopharm 2008, 68(1):112-128,

30. Lim YT, Cho MY, Lee JM, Chung SJ, Chung BH: Simultaneous intracellular delivery of targeting antibodies and functional nanoparticles with engineered protein G system. Biomaterials 2009, 30(6):1197-1204.

31. Hatakeyama H, Akita H, Ishida E, Hashimoto K, Kobayashi H, Aoki T, Yasuda J, Obata K, Kikuchi H, Ishida T, et al: Tumor targeting of doxorubicin by anti-MT1-MMP antibody-modified PEG liposomes. Int J Pharm 2007, 342(1-2):194-200.

32. Kirpotin DB, Drummond DC, Shao Y, Shalaby MR, Hong K, Nielsen UB, Marks JD, Benz CC, Park JW: Antibody targeting of long-circulating lipidic nanoparticles does not increase tumor localization but does increase internalization in animal models. Cancer Res 2006, 66(13):6732-6740.

33. Liu P, Wang B, Weili Qiao JL: Multi-anticancer drugs encapsulated in the micelle: a novel chemotherapy to cancer. Med Hypotheses 2008 , 71(3):379-381.

34. Matsumura Y: Basic Aspects and Clinical Trials of Micelle Carrier System. MEMS, NANO and Smart Systems, ICMENS 2004: 25-27 Aug. 2004610.
35. Kataoka K, Harada A, Nagasaki Y: Block copolymer micelles for drug delivery: design, characterization and biological significance. Adv Drug Deliv Rev 2001, 47(1):113-131.

36. Hwang MJ, Suh JM, Bae YH, Kim SW, Jeong B: Caprolactonic poloxamer analog: PEG-PCL-PEG. Biomacromolecules 2005, 6(2):885-890.

37. Liu J, Xiao Y, Allen C: Polymer-drug compatibility: a guide to the development of delivery systems for the anticancer agent, ellipticine. $J$ Pharm Sci 2004, 93(1):132-143.

38. Liggins RT, Burt HM: Polyether-polyester diblock copolymers for the preparation of paclitaxel loaded polymeric micelle formulations. Adv Drug Deliv Rev 2002, 54(2):191-202.

39. Yallapu MM, Jaggi M, Chauhan SC: beta-Cyclodextrin-curcumin selfassembly enhances curcumin delivery in prostate cancer cells. Colloids Surf B Biointerf 2010, 79(1):113-125.

40. Yallapu MM, Jaggi M, Chauhan SC: Poly(beta-cyclodextrin)/Curcumin SelfAssembly: A Novel Approach to Improve Curcumin Delivery and its Therapeutic Efficacy in Prostate Cancer Cells. Macromol Biosci , 10.1002/ mabi.201000084.

41. Yallapu MM, Jaggi M, Chauhan SC: Fabrication of curcumin encapsulated PLGA nanoparticles for improved therapeutic effects in metastatic cancer cells. J Colloid Interf Sci 2010.

42. Yasugi K, Nagasaki Y, Kato M, Kataoka K: Preparation and characterization of polymer micelles from poly(ethylene glycol)-poly(D,L-lactide) block copolymers as potential drug carrier. J Control Release 1999, 62(12):89-100.

43. Solomon $\mathrm{CH}$, Pho LN, Burt RW: Current status of genetic testing for colorectal cancer susceptibility. Oncology 2002, 16(2):161-171.

44. Yoo HS, Park TG: Biodegradable polymeric micelles composed of doxorubicin conjugated PLGA-PEG block copolymer. J Control Release 2001, 70(1-2):63-70.

45. Jette KK, Law D, Schmitt EA, Kwon GS: Preparation and drug loading of poly(ethylene glycol)-block-poly(epsilon-caprolactone) micelles through the evaporation of a cosolvent azeotrope. Pharm Res 2004, 21(7):1184-1191.

46. Chen C, Yu CH, Cheng YC, Yu PH, Cheung MK: Biodegradable nanoparticles of amphiphilic triblock copolymers based on poly(3hydroxybutyrate) and poly(ethylene glycol) as drug carriers. Biomaterials 2006, 27(27):4804-4814

47. La SB, Okano T, Kataoka K: Preparation and characterization of the micelle-forming polymeric drug indomethacin-incorporated poly (ethylene oxide)-poly(beta-benzyl L-aspartate) block copolymer micelles. J Pharm Sci 1996, 85(1):85-90.

48. Chen $\mathrm{C}, \mathrm{Yu} \mathrm{CH}$, Cheng YC, Yu PH, Cheung MK: Micelle formation and solgel transition behavior of comb-like amphiphilic poly((PLGA-b-PEG)MA) copolymers. J Polym Sci Part A Polym Chem 2008, 46(6):1954-1963.

49. Park I, Lee H, Chang T, Kim SW, Lee DS: Poly(D,L-lactic acid-co-glycolic acid)-b-poly(ethylene glycol)-b-poly ( $D, L-$ lactic acid-co-glycolic acid) triblock copolymer and thermoreversible phase transition in water. $J$ Biomed Mater Res 2002, 61(2):188-196.

50. Lee SJ, Han BR, Park SY, Han DK, Kim SC: Sol-gel transition behavior of biodegradable three-arm and four-arm star-shaped PLGA-PEG block copolymer aqueous solution. J Polym Sci Part A Polym Chem 2006, 44(2):888-899.

51. Kim HK, Park TG: Surface Stabilization of Diblock PEG-PLGA Micelles by Polymerization of N-Vinyl-2-pyrrolidone. Macromol Rapid Commun 2002, 23(1):26-31.

52. Lee H, Ahn CH, Park TG: Poly[lactic-co-(glycolic acid)]-Grafted Hyaluronic Acid Copolymer Micelle Nanoparticles for Target-Specific Delivery of Doxorubicin. Macromol Biosci 2009, 9(4):336-42.

53. Kim SH, Jeong JH, Chun KW, Park TG: Target-Specific Cellular Uptake of PLGA Nanoparticles Coated with Poly(l-lysine)-Poly(ethylene glycol)Folate Conjugate. Langmuir 2005, 21(19):8852-8857.

54. Vinogradov SV, Zeman AD, Batrakova EV, Kabanov AV: Polyplex Nanogel formulations for drug delivery of cytotoxic nucleoside analogs. J Control Release 2005, 107(1):143-157.

55. Vinogradov SV, Bronich TK, Kabanov AV: Nanosized cationic hydrogels for drug delivery: preparation, properties and interactions with cells. Adv Drug Deliv Rev 2002, 54(1):135-147.

56. Galmarini CM, Warren G, Kohli E, Zeman A, Mitin A, Vinogradov SV: Polymeric nanogels containing the triphosphate form of cytotoxic 
nucleoside analogues show antitumor activity against breast and colorectal cancer cell lines. Mol Cancer Ther 2008, 7(10):3373-3380.

57. Sahu A, Kasoju N, Bora U: Fluorescence Study of the Curcumin-Casein Micelle Complexation and Its Application as a Drug Nanocarrier to Cancer Cells. Biomacromolecules 2008, 9(10):2905-2912.

58. Kim SC, Kim DW, Shim YH, Bang JS, Oh HS, Wan Kim S, Seo MH: In vivo evaluation of polymeric micellar paclitaxel formulation: toxicity and efficacy. J Control Release 2001, 72(1-3):191-202.

59. Jubo L, Yuehua $X$, Christine A: Polymer-drug compatibility: A guide to the development of delivery systems for the anticancer agent, ellipticine. J Pharm Sci 2004, 93(1):132-143.

60. Shuai X, Merdan T, Schaper AK, Xi F, Kissel T: Core-Cross-Linked Polymeric Micelles as Paclitaxel Carriers. Bioconj Chem 2004, 15(3):441-448.

61. Shuai X, Ai H, Nasongkla N, Kim S, Gao J: Micellar carriers based on block copolymers of poly(epsilon-caprolactone) and poly(ethylene glycol) for doxorubicin delivery. J Control Release 2004, 98(3):415-426.

62. Wang Y-C, Tang L-Y, Sun T-M, Li C-H, Xiong M-H, Wang J: Self-Assembled Micelles of Biodegradable Triblock Copolymers Based on Poly(ethyl ethylene phosphate) and Poly( $\varepsilon$-caprolactone) as Drug Carriers. Biomacromolecules 2008, 9(1):388-395.

63. Wang J, Woodcock SE, Buck SM, Chen C, Chen Z: Different SurfaceRestructuring Behaviors of Poly(methacrylate)s Detected by SFG in Water. J Am Chem Soc 2001, 123(38):9470-9471.

64. Licciardi M, Craparo EF, Giammona G, Armes SP, Tang Y, Lewis AL: in vitro biological evaluation of folate-functionalized block copolymer micelles for selective anti-cancer drug delivery. Macromol Biosci 2008, 8(7):615-626.

65. Kim SH, Jeong JH, Lee SH, Kim SW, Park TG: LHRH Receptor-Mediated Delivery of siRNA Using Polyelectrolyte Complex Micelles SelfAssembled from siRNA-PEG-LHRH Conjugate and PEI. Bioconj Chem 2008, 19(11):2156-2162.

66. Lee H, Hu M, Reilly RM, Allen C: Apoptotic Epidermal Growth Factor (EGF)-Conjugated Block Copolymer Micelles as a Nanotechnology Platform for Targeted Combination Therapy. Mol Pharm 2007, 4(5):769-781

67. Bae Y, Fukushima S, Harada A, Kataoka K: Design of environment-sensitive supramolecular assemblies for intracellular drug delivery: polymeric micelles that are responsive to intracellular $\mathrm{pH}$ change. Angew Chem Int 2003, 42(38):4640-4643.

68. Bae $Y$, Nishiyama N, Kataoka K: In vivo antitumor activity of the folateconjugated $\mathrm{pH}$-sensitive polymeric micelle selectively releasing adriamycin in the intracellular acidic compartments. Bioconjug Chem 2007, 18(4):1131-1139.

69. Kim D, Lee ES, Park K, Kwon IC, Bae YH: Doxorubicin loaded pH-sensitive micelle: antitumoral efficacy against ovarian A2780/DOXR tumor. Pharm Res 2008, 25(9):2074-2082.

70. Kyung Taek Oh ESL: Cancer-associated pH-responsive tetracopolymeric micelles composed of poly(ethylene glycol)-b-poly(L-histidine)-b-poly(Llactic acid)-b-poly(ethylene glycol). Polym Adv Technol 2008, 19(12):1907-1913.

71. Lee ES, Gao Z, Kim D, Park D, Kwon IC, Bae YH: Super pH-sensitive multifunctional polymeric micelle for tumor pHe specific TAT exposure and multidrug resistance. J Control Release 2008, 129(3):228-236

72. Yoo HS, Lee EA, Park TG: Doxorubicin-conjugated biodegradable polymeric micelles having acid-cleavable linkages. I Control Release 2002, 82(1):17-27.

73. Lee Y, Park SY, Mok H, Park TG: Synthesis, Characterization, Antitumor Activity of Pluronic Mimicking Copolymer Micelles Conjugated with Doxorubicin via Acid-Cleavable Linkage. Bioconj Chem 2008, 19(2):525-531.

74. Burt HM, Zhang $X$, Toleikis P, Embree L, Hunter WL: Development of copolymers of poly(D,L-lactide) and methoxypolyethylene glycol as micellar carriers of paclitaxel. Colloids Surf B Biointer 1999, 16(1-4):161-171

75. Chen H, Kim S, He W, Wang H, Low PS, Park K, Cheng JX: Fast Release of Lipophilic Agents from Circulating PEG-PDLLA Micelles Revealed by in Vivo Förster Resonance Energy Transfer Imaging. Langmuir 2008, 24(10):5213-5217.

76. O'Reilly RK, Hawker CJ, Wooley KL: Cross-linked block copolymer micelles: functional nanostructures of great potential and versatility. Chem Soc Rev 2006, 35(11):1068-1083
77. Bontha S, Kabanov AV, Bronich TK: Polymer micelles with cross-linked ionic cores for delivery of anticancer drugs. J Control Release 2006, 114(2):163-174.

78. Chan Y, Wong T, Byrne F, Kavallaris M, Bulmus V: Acid-Labile Core CrossLinked Micelles for $\mathrm{pH}$-Triggered Release of Antitumor Drugs. Biomacromolecules 2008, 9(7):1826-1836.

79. van der Burgh S, de Keizer A, Stuart CMA: Complex Coacervation Core Micelles. Colloidal Stability and Aggregation Mechanism. Langmuir 2004, 20(4):1073-1084

80. Stuart CMA, Besseling NAM, Fokkink RG: Formation of Micelles with Complex Coacervate Cores. Langmuir 1998, 14(24):6846-6849.

81. Nishiyama N, Kataoka K: Current state, achievements, and future prospects of polymeric micelles as nanocarriers for drug and gene delivery. Pharmacol Ther 2006, 112(3):630-648.

82. Harada A, Kataoka K: Novel polyion complex micelles entrapping enzyme molecules in the core: preparation of narrowly-distributed micelles from lysozyme and poly(ethylene glycol)-poly(aspartic acid) block copolymer in aqueous medium. Macromol 1998, 31(2):288-294.

83. Aoyagi T, Sugi K, Sakurai Y, Okano T, Kataoka K: Peptide drug carrier: studies on incorporation of vasopressin into nano-associates comprising poly(ethylene glycol)-poly(-aspartic acid) block copolymer. Colloids Surf $B$ Biointerf 1999, 16(1-4):237-242.

84. Yokoyama M, Miyauchi M, Yamada N, Okano T, Sakurai Y, Kataoka K: Characterization and anticancer activity of the micelle-forming polymeric anticancer drug adriamycin-conjugated poly(ethylene glycol)poly(aspartic acid) block copolymer. Cancer Res 1990, 50:1693-1700.

85. Gao Z, Fain HD, Rapoport N: Ultrasound-enhanced tumor targeting of polymeric micellar drug carriers. Mol Pharm 2004, 1(4):317-330.

86. Marin A, Sun H, Husseini GA, Pitt WG, Christensen DA, Rapoport NY: Drug delivery in pluronic micelles: effect of high-frequency ultrasound on drug release from micelles and intracellular uptake. $J$ Control Release 2002, 84(1-2):39-47.

87. Namdeo M, Sutanjay S, Tankhiwale R, Bajpai M, Yallapu MM, Bajpai SK: Magnetic Nanoparticles for Drug Delivery Applications. J Nanosci Nanotech 2008, 8(7):3247-3271.

88. McCarthy JR, Weissleder R: Multifunctional magnetic nanoparticles for targeted imaging and therapy. Adv Drug Deliv Rev 2008, 60(11):1241-1251.

89. Bertorelle F, Wilhelm C, Roger J, Gazeau F, Menager C, Cabuil V: Fluorescence-modified superparamagnetic nanoparticles: intracellular uptake and use in cellular imaging. Langmuir 2006, 22(12):5385-5391.

90. Cinteza LO, Ohulchanskyy TY, Sahoo Y, Bergey EJ, Pandey RK, Prasad PN: Diacyllipid micelle-based nanocarrier for magnetically guided delivery of drugs in photodynamic therapy. Mol Pharm 2006, 3(4):415-423.

91. Guo R, Zhang L, Qian H, Li R, Jiang X, Liu B: Multifunctional nanocarriers for cell imaging, drug delivery, and near-IR photothermal therapy. Langmuir 2010, 26(8):5428-5434.

92. Guthi JS, Yang SG, Huang G, Li S, Khemtong C, Kessinger CW, Peyton M, Minna JD, Brown KC, Gao J: MRI-visible micellar nanomedicine for targeted drug delivery to lung cancer cells. Mol Pharm 2010, 7(1):32-40.

93. Pradhan P, Giri J, Rieken F, Koch C, Mykhaylyk O, Doblinger M, Banerjee R, Bahadur D, Plank C: Targeted temperature sensitive magnetic liposomes for thermo-chemotherapy. J Control Release 142(1):108-121.

94. Rubio-Retama J, Zafeiropoulos NE, Serafinelli C, Rojas-Reyna R, Voit B, Cabarcos EL, Stamm M: Synthesis and characterization of thermosensitive PNIPAM microgels covered with superparamagnetic gamma-Fe2O3 nanoparticles. Langmuir 2007, 23(20):10280-10285.

95. Shubayev VI, Pisanic TR, Jin S: Magnetic nanoparticles for theragnostics. Adv Drug Deliv Rev 2009, 61(6):467-477.

96. Wang L, Yang Z, Zhang Y, Wang L: Biofunctional nanoparticles with magnetization and luminescence. J Phys Chem C 2009, 113(10):3955-3959.

97. Jain TK, Foy SP, Erokwu B, Dimitrijevic S, Flask CA, Labhasetwar V: Magnetic resonance imaging of multifunctional pluronic stabilized iron-oxide nanoparticles in tumor-bearing mice. Biomaterials 2009, 30(35):6748-6756.

doi:10.1186/1757-2215-3-19

Cite this article as: Yallapu et al: Scope of nanotechnology in ovarian cancer therapeutics. Journal of Ovarian Research 2010 3:19. 